\title{
Resource Development
}

National Cancer Institute

\section{Source}

National Cancer Institute. Resource Development. NCI Thesaurus. Code C19441.

Development of a new technology through the feasibility-testing stage to the level of a robust experimental or analytical tool. Development and maintenance of specialized specimen resources to facilitate basic and diagnostic cancer research, including tissue banks, tissue procurement, networks, and DNA banks. Development of new tissue processing techniques and new reagents to better preserve critical molecular components to facilitate cancer diagnostic research. Informatics support of tissue resources and diagnostic cancer research. (from Guide for Applicants: Genome Research Resource Grants (P41), Draft 1/24/95) 